\title{
BMJ Open Association between functional disability and depressive symptoms among older adults in rural China: a cross-sectional study
}

\author{
Jian Rong, ${ }^{1}$ Xueqin Wang, ${ }^{2}$ Yanhong Ge, ${ }^{3}$ Guimei Chen, ${ }^{3}$ Hong Ding (D) ${ }^{3}$
}

To cite: Rong J, Wang X, Ge Y, et al. Association between functional disability and depressive symptoms among older adults in rural China: a cross-sectional study. BMJ Open 2021;11:e047939. doi:10.1136/ bmjopen-2020-047939

- Prepublication history and additional supplemental material for this paper are available online. To view these files, please visit the journal online (http://dx.doi.org/10.1136/ bmjopen-2020-047939)

Received 14 December 2020 Accepted 08 December 2021

A) Check for updates

C Author(s) (or their employer(s)) 2021. Re-use permitted under CC BY-NC. No commercial re-use. See rights and permissions. Published by BMJ.

${ }^{1}$ Department of Scientific Research, The Second Hospital of Anhui Medical University, Hefei, Anhui, China

${ }^{2}$ Department of Medical Engineering, The Second Hospital of Anhui Medical University, Hefei, Anhui, China ${ }^{3}$ Department of Health Service Management, Anhui Medical University, Hefei, Anhui, China

Correspondence to

Dr Hong Ding;

dinghong2003@126.com

\section{ABSTRACT}

Objectives The aim of this study was to explore the relationship between functional disability and depressive symptoms, focusing on whether an interaction exists between functional disability, demographic characteristics and depressive symptoms among older adults in rural China.

Design A cross-sectional study using multistage, stratified random sampling.

Setting Data from 18 villages in Anhui Province of China between January to July 2018.

Participants 3491 Chinese participants aged 60 and over. Primary and secondary outcome measures The 30-item Geriatric Depression Scale and WHO Disability Assessment Schedule 2.0 were used to evaluate depressive symptoms and functional disability, respectively. Data were analysed using SPSS statistics V.25.0 program with $\chi^{2}$ test, Mann-Whitney U test, binary logistic regression analysis and classification and regression tree (CART) model.

Results The prevalence of depressive symptoms in 3336 interviewed older people was $52.94 \%$. After adjustment, subjects who had problems in mobility domain (adjusted OR (AOR) 1.842, 95\% Cl 1.503 to 2.258), getting along domain (AOR 1.616, 95\% Cl 1.299 to 2.010), life activities domain (AOR $1.683,95 \% \mathrm{Cl} 1.370$ to 2.066) and participation domain (AOR $3.499,95 \% \mathrm{Cl} 2.385$ to 4.987) had an increased depressive symptoms risk. However, cognition domain (AOR $0.785,95 \% \mathrm{Cl} 0.647$ to 0.953) negatively correlated with depressive symptoms. Additionally, the CART model showed that those who had problems in mobility domain, getting along domain and were unemployed, the possibility of having depressive symptoms was the highest.

Conclusions More attention should be paid to unemployed older adults, and those with problems in participation, life activities, getting along and mobility and no problems in cognition to maintain a good psychological state.

\section{INTRODUCTION}

With the development of China's economy and the change of population structure, China is witnessing an escalating process of population ageing. By the end of 2019, China had a population of 253.88 million
Strengths and limitations of this study

- The sample response rate of is high, and the largescale survey results with high response rate are very robust.

- The classification and regression tree model was conducted, which can not only explore the interaction between multivariables, but also be displayed by intuitive tree graph.

- This is the first study to explore the interaction between functional disability and demographic factors related to depressive symptoms in the Chinese population.

- Because of the characteristics of a cross-sectional study, inferring the causality between functional disability and depressive symptoms is limited.

- The extension of the research results to other regions or other countries is limited.

aged 60 or above, accounting for $18.1 \%$ of the total population. ${ }^{1}$ It was predicted that the proportion of older adults aged 60 and over will reach $35.1 \%$ of the total population by $2050 .{ }^{2}$ With this rapid growth, the mental disorders among older adults has attracted an increasing number of policy makers and health managers in China. ${ }^{3}$

Depressive symptom is a common mental disorder among older adults and has developed into an important public health problem. ${ }^{4}$ Patients with depressive symptom are characterised by listlessness, lack of interest in normal life and low comprehension and accompanied by fatigue, weight change and inattention. ${ }^{5}$ The global prevalence of depressive symptom in the older adults varies between $10 \%$ and $20 \%$ and also varies between different demographic characteristics. ${ }^{67}$ Our previous meta-analysis found that the prevalence of depressive symptoms among the adults aged 60 and older in China from 2010 to 2019 was $25.55 \%$, especially in rural areas, the prevalence reached $31.02 \%{ }^{8}$ Studies have shown that depressive symptoms 
have increased the risk of death and suicide, and the cost of treatment also putting tremendous financial burden on families and the society. ${ }^{9}$ A study found that the total cost of caring for older adults with depressive symptom reached 3748 euros for half a year. ${ }^{10}$ Furthermore, older adults with depressive symptom were at risk for dementia, coronary heart disease, stroke, cancer and suicide. ${ }^{11}$ Additionally, it was predicted that depressive symptom will become the second leading cause of disability worldwide by $2020{ }^{12}$ Therefore, it is of great practical significance to identify factors related to depressive symptoms for centralised screening and subsequent development of preventive interventions. Studies have indicated that the complex interaction of social, psychological and biological factors can lead to depressive symptoms. ${ }^{13} 14$ Many bad lifestyles also increase the risk of depressive symptoms. ${ }^{15} 16$ Previous study also found that social participation plays a protective role against depressive symptoms among older adults. ${ }^{17}$

Studies have shown that functional disability of the older adults is a major public health problem in many countries, including China. ${ }^{18}$ Functional disability in the older adults has not only a negative impact on independent living ability but also reduces the quality of life. ${ }^{19}$ Approximately $15.0 \%$ of the world's population have different degrees of functional disability. ${ }^{20}$ There are differences in functional disability prevalence among countries. A cross-sectional survey in Morocco showed that the overall functional disability prevalence of older adults was $9.5 \% .{ }^{21}$ A Dutch study showed that the prevalence of functional disability in the older adults was about $22 \%{ }^{22}$ According to the data analysis of Behavioral Risk Factors Surveillance System in 2016, the prevalence of mobile disability was $26.9 \%$ in the older adults. ${ }^{23}$ The prevalence of functional disability of older adults in Asia varies from $16.2 \%$ in China to $55.7 \%$ in India. ${ }^{24}$ Several domestic and foreign studies have found that increasing age, and weakness were associated with functional disability. 2526

Previous studies have shown that there was an important correlation between functional disability and depressive symptoms in the older adults. ${ }^{27-29}$ Nyunt $e t$ al found that the improvement in the levels and remission of depressive symptoms were associated with improvement in functional ability among community-living older adults who were treated for depressive symptoms in a primary care setting in Singapore. ${ }^{27}$ A study in Taiwan found that functional disability may contribute to subsequent depressive symptoms by reducing activity and social support. ${ }^{28}$ Another study found that older adults with problems with daily living disability were more likely to develop depressive symptoms. ${ }^{29}$

However, most existing studies were based on the Western population and mainly aimed at revealing the related factors of depressive symptoms among older adults. ${ }^{13-17}$ At present, epidemiological evidence for the association between functional disability and depressive symptoms continue to remain insufficient, especially for the vulnerable group, that is, the older adults in rural areas in China. A comprehensive understanding of factors related to depressive symptoms is of great theoretical and practical significance for the formulation of specific and appropriate intervention strategies to reduce the occurrence of depressive symptoms in the older adults. It can provide empirical reference for China's rural areas and other developing countries to further improve the mental health of rural area-based older adults and promote grassroots public health services. In this context, this study explored the relationship between functional disability and depressive symptoms of the older adults in the rural areas of Anhui Province, China, with a focus on whether there is an interaction between functional disability, demographic characteristics and depressive symptoms.

\section{METHODS}

\section{Study design and sample}

To obtain a better representative sample, this crosssectional survey was conducted using a multi-stage sampling survey method from January 2018 to July 2018. According to the geographical environment factors, Anhui Province is usually divided into three regions: northern Anhui, central Anhui and southern Anhui. First, we selected three prefecture-level cities from the 16 prefecture-level cities in Anhui Province: Fuyang (North); Hefei (central, the capital of Anhui provincial) and Anqing (South). Second, in each prefecture-level city, one county was randomly selected. Next, in each selected county, two townships were randomly selected. A total of six townships were finally selected. At the last stage, we randomly selected three villages in each selected township, and a total of 18 villages were identified as the survey sites.

According to the data of local household registration system, 50 poor households (the per capita net income of rural households in China was less than ¥2736 in 2013) were randomly selected from each selected village based on the list of poor households, and 75 non-poor households were randomly selected from the neighbours of poor households. The adults (aged $\geq 60$ ) from poor and non-poor households were selected as the survey participants. With the assistance of local community workers and village doctors, each subject was investigated in the form of a household survey, and a face-to-face questionnaire survey was conducted by the graduate students from Anhui Medical University who had received unified training. The purpose and procedures of the study were dictated to all respondents, and confidentiality through verbal informed consent prior to participating in the study. Persons aged 60 and over and living in their residence for at least 1 year participated in the survey. Individuals with cognitive impairment, with language communication disorders, who were deaf, and with lack of access during the survey were excluded.

A total of 3491 older adults participated in the survey, and 155 older adults who were exhausted or occupied and could not complete the questionnaire were 
excluded. Finally, 3336 older adults were included for further analyses, with an effective response rate of $95.56 \%$ (3336/3491).

\section{Assessment instruments}

Thirty-item Geriatric Depression Scale

Depressive symptoms were evaluated using 30-item Geriatric Depression Scale (GDS-30), which was specifically designed for screening depressive symptoms in older adults. ${ }^{30}$ This scale comprises 30 items; each item is dichotomised into two categories: 'Yes' or 'No'. Among the 30 items, 10 items were scored in reverse order (1 point for 'No' and 0 point for 'Yes'), and the remaining were scored in positive order ( 1 point for 'Yes' and 0 point for 'No'). The total score ranged from 0 to 30 points, and the clinical boundary was 10 points. An individual with a GDS-30 score $\leq 10$ was regarded as non-depressive symptoms and with a GDS-30 score $\geq 11$ as depressive symptoms. ${ }^{31}$ Studies have confirmed that GDS-30 had a high sensitivity $(70.6 \%)$ and high specificity $(70.1 \%)$ in a Chinese population aged 60 and over. ${ }^{32}$ In this study, the internal consistency of GDS-30 is as indicated by the Cronbach's $\alpha$ score of 0.890 .

\section{WHO Disability Assessment Schedule (WHODAS 2.0)}

WHO Disability Assessment Schedule (WHODAS 2.0) was employed to examine the functional disability of the respondents. The scale consists of 36 items, including six domains: (1) cognition domain-understanding and communicating, (2) mobility domain-moving and getting around, (3) self-care domain-attending to one's hygiene, dressing, eating and staying alone, (4) getting along domain-interacting with other people, (5) life activities domain-domestic responsibilities, leisure, work and school, and (6) participation domain-joining in community activities, participating in society. Each item is scored on the Likert 5 scale $(1=$ no difficulty, $2=$ mild difficulty, $3=$ moderate difficulty, $4=$ severe difficulty, $5=$ extreme difficulty). According to the scale instruction manual, ${ }^{33}$ we recoded the scores of each domain, calculated each evaluation dimension and the overall score, and converted the original score to correspond to points $0-100(0=$ no disability, $100=$ complete disability $)$. Based on the International Classification of Functioning disability and health criteria, each evaluation dimension and overall disability level was evaluated, that is, $<4$ points are classified as no problem disability status and $>4$ points as mild or above disability status. ${ }^{34}$ The internal consistency of the schedule is as indicated by the Cronbach's $\alpha$ score of 0.967 in this study.

\section{Demographic characteristics}

The survey also collected demographic characteristic variables. These variables included gender (male, female), age (60-69, 70-79, $\geq 80)$, educational level (illiterate and primary school and above), employment status (unemployed, employed), living style (living alone, living with spouse, others refer to living with children or other relatives), region (northern, central, southern), poverty (families whose annual per capita income was lower than the national poverty line need to be recognised by the Poverty Alleviation Office and other departments). Simultaneously, we also investigated the variables related to health status, including whether they had physical discomfort during the previous 2 weeks (Yes, No), chronic disease diagnosed by doctors (No, Yes), or were hospitalised in the previous year (Yes, No).

\section{Statistical analysis}

Descriptive statistics were used to assess the sample demographic characteristics (frequency and percentages). Median and IQRs were measured as the data showed skewed distribution. Mann-Whitney U tests were employed to compare the functional disability of participants with different depressive symptoms. The $\chi^{2}$ tests were also employed to examine the difference between different depressive symptoms groups.

Binary logistic regression analysis with an enter method was used to analyse the relationship between functional disability and depressive symptoms after adjusting for potential covariates (including gender, age, educational level, employment status, living style, region, poverty, physical discomfort, chronic diseases and hospitalisation). According to the criteria of tolerance and variance inflation factor, there was no multicolinearity between independent variables (online supplemental table S1 and S2). For functional disability variables, those who had no problem in each dimension were grouped as the reference group in a logistic regression model. For other variables, participants who were male, aged 60-69 years, illiterate, unemployed, living alone, belonging to the northern region, poor, had physical discomfort, had no chronic disease, hospitalised were grouped as reference. The results of the binary logistic regression analysis were expressed with the adjusted OR (AOR) and associated $95 \%$ CI.

To further investigate the interaction between functional disability, demographic factors and depressive symptoms, a classification and regression tree model (CART) was used. This model is a novel analysis method, which can automatically judge and classify according to the significance of the test, optimal segment many types of variables and samples, and show the interaction between different variables through the tree diagram. It can also overcome the colinearity problem that exists in the traditional analysis method and investigate the complex combination or interaction between the factors and variables neglected in the traditional analysis method. ${ }^{35}$ The $\chi^{2}$ automation interaction detector algorithm was used to screen variables and include statistically significant variables in univariate analysis. Additionally, to fully mine the interaction between variables and keep the tree structure relatively simple, the minimum cases in the parent node and child node of the model parameters were set 400 and 200 , respectively, and the maximum growth depth was 3 . 
Table 1 Demographic characteristics of respondents, n (\%)

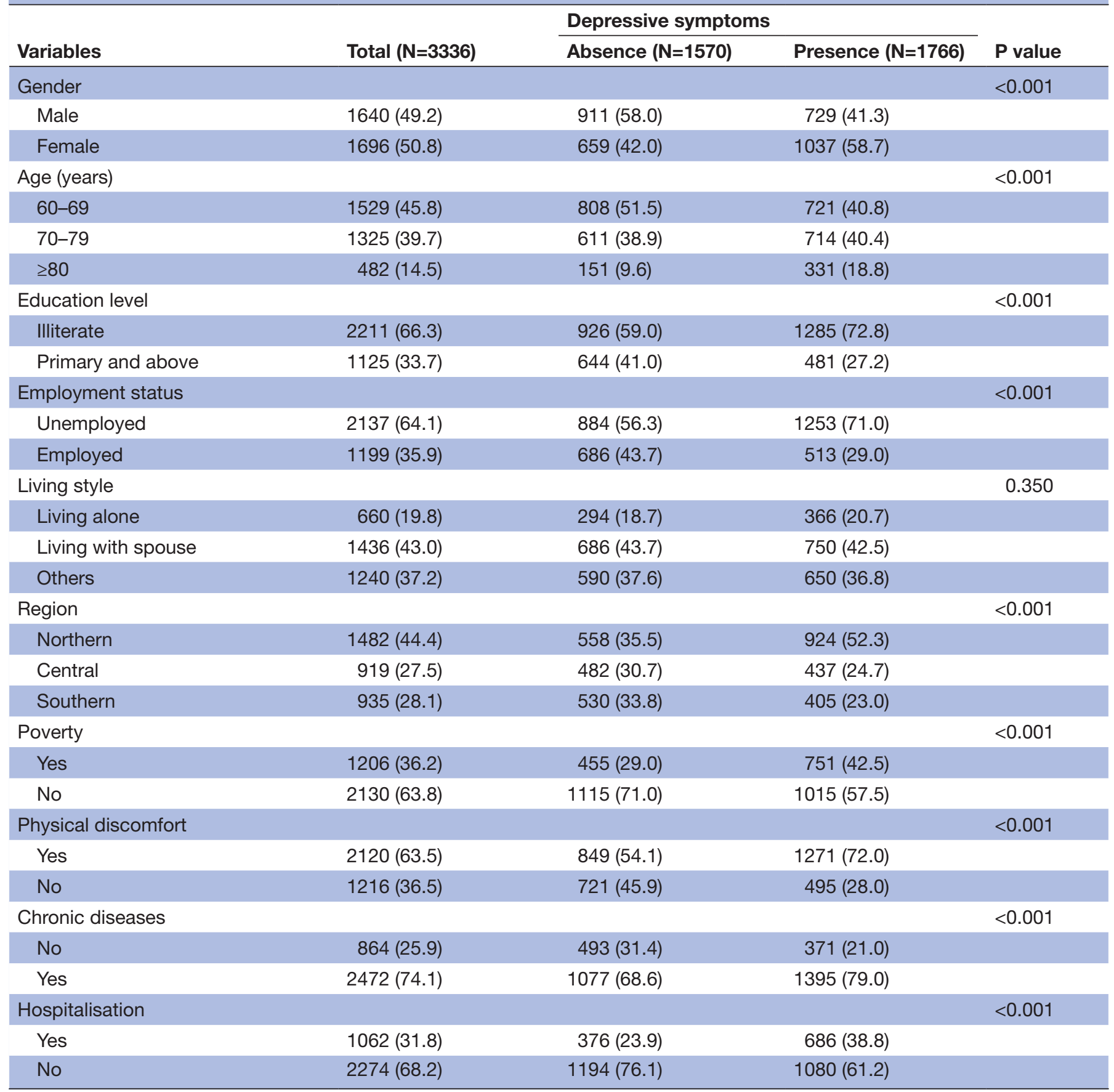

All data were analysed using SPSS statistics software, V.25.0 (IBM). A two-tailed $\mathrm{p}<0.05$ was considered to be statistically significant.

\section{Patients and public involvement}

Neither patients nor the public were involved in the development of the methodology for the current study.

\section{RESULTS}

Sample characteristics

The demographic characteristics of respondents is shown in table 1. Age distribution of 3336 respondents included in the analysis ranged from 60 to 97 years, and median (IQR) was 70 (65.25-76). Most of the respondents were female $(50.8 \%)$, illiterate $(66.3 \%)$, unemployed $(64.1 \%)$, living with spouse $(43.0 \%)$, belonging to the northern (44.4\%), non-poor (63.8\%), had physical discomfort (63.5\%), had chronic diseases $(74.1 \%)$ and hospitalised (68.2\%). Except for variable of living style, there was a statistically significant difference in depressive symptoms among demographic variables (all $\mathrm{p}<0.05)$.

\section{Overview of depressive symptoms}

Among the 3,336 older adults people participants, 1766 $(52.94 \%)$ had depressive symptoms. The average score of 


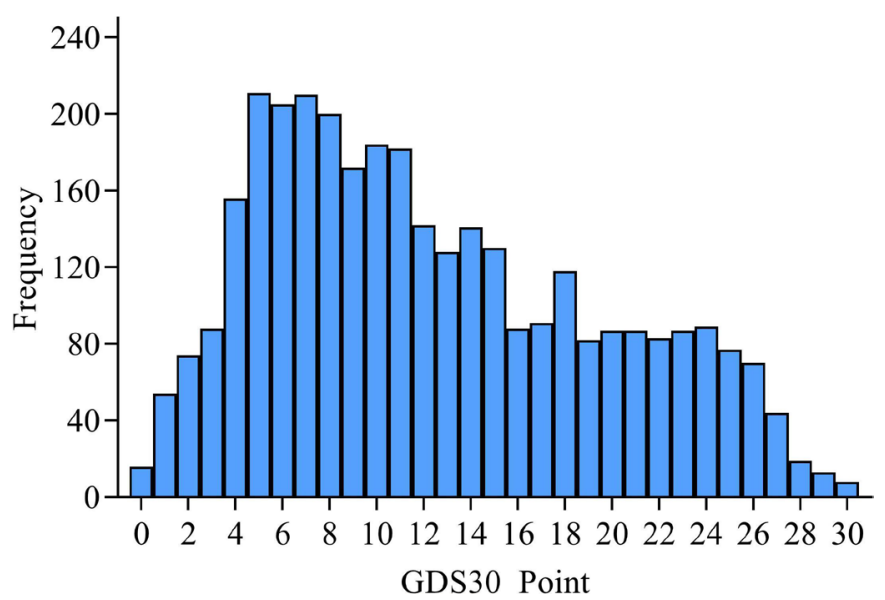

Figure 1 GDS-30 score distribution of the subjects.GDS-30, 30-item Geriatric Depression Scale.

depressive symptoms of participants was 12.4 points, with median (IQR) 11 (7-18) points and the high frequency scores of GDS-30 were concentrated between 4 and 15 points (figure 1).

\section{Distribution of functional disability among participants}

The distribution of functional disability among participants with different depressive symptoms is shown in table 2. The subjects had the highest score in the dimension of living activities (31.91 points) and the lowest score in self-care (11.33 points). Compared with the group without depressive symptoms, the subjects with depressive symptoms had higher scores in domains of cognition, mobility, self-care, getting along, life activities and participation (higher score=greater extent of disabilities) and the difference between the two groups was statistically significant (all $\mathrm{p}<0.05)$.

\section{Association between functional disability and depressive symptoms}

The association between functional disability and depressive symptoms is shown in table 3 . Binary logistic regression was used with the presence of depressive symptoms (absence $=1$, presence $=2$ ) as the dependent variable, and each dimension of disability status (had no problem as the reference group) was taken as the independent variable. After adjustment, the results showed that compared with the reference group, subjects had problems in domains of mobility (AOR 1.842, 95\% CI 1.503 to 2.258), getting along (AOR1.616, 95\% CI 1.299 to 2.010), life activities (AOR1.683, 95\% CI 1.370 to 2.066) and participation (AOR3.499, 95\% CI 2.385 to 4.987 ) that were related risk factors for depressive symptoms (all $\mathrm{p}<0.05)$. However, problems in cognition dimension (AOR0.785, 95\% CI 0.647 to 0.953 ) was a protective factor for depressive symptoms $(\mathrm{p}=0.015)$.

\section{Results of CART model}

CART model was conducted with depressive symptom (absence $=1$, presence $=2$ ) as the dependent variable and the dimension of functional disability and demographic characteristics as the classification nodes. The results are shown in figure 2. The model includes three depth, 13 nodes, including 7 terminal nodes. Depressive symptoms were mainly concerned with domains of mobility, participation, life activities, getting along and also employment status. Among which, mobility domain was the first split factor associated with depressive symptoms.

Among participants who had no problem in mobility domain (node 1) but in participation domain (node 4) and life activities domain (node 8) had a high rate of having depressive symptoms than those with no problem in life activities domain (node 7).

For those with no problems in mobility domain (node 1) and participation domain (node 3), the proportion $(10.9 \%)$ of developing depressive symptoms was the least.

Those who had problems in mobility domain(node 2), getting along domain(node 6) and were unemployed (node 12), the possibility of having depressive symptoms was the highest (node 12).

\section{DISCUSSION}

This study explored the association between functional disability, demographic characteristics and depressive symptoms among older adults in rural Anhui, China. The results showed that a relationship between functional

Table 2 Distribution of functional disability among participants

\begin{tabular}{|c|c|c|c|c|c|c|c|}
\hline \multirow{3}{*}{$\begin{array}{l}\text { Functional } \\
\text { disability } \\
\text { domains }\end{array}$} & \multirow{2}{*}{\multicolumn{2}{|c|}{ Total $(\mathrm{N}=3336)$}} & \multicolumn{4}{|c|}{ Depressive symptoms } & \multirow[b]{3}{*}{ P value } \\
\hline & & & \multicolumn{2}{|c|}{ Absence $(\mathrm{N}=1570)$} & \multicolumn{2}{|c|}{ Presence $(\mathrm{N}=1766)$} & \\
\hline & GM & Median (IQR) & GM & Median (IQR) & GM & Median (IQR) & \\
\hline Mobility & 26.57 & $18.75(0.00-43.75)$ & 15.33 & $0.00(0.00-25.00)$ & 36.57 & 37.50 (12.50-56.25) & $<0.001$ \\
\hline Self-care & 11.33 & $0.00(0.00-10.00)$ & 4.87 & $0.00(0.00-0.00)$ & 17.07 & $0.00(0.00-40.00)$ & $<0.001$ \\
\hline Life activities & 31.91 & $30.00(0.00-40.00)$ & 19.83 & $0.00(0.00-40.00)$ & 42.64 & $40.00(30.00-50.00)$ & $<0.001$ \\
\hline Participation & 29.39 & 25.00 (12.50-41.67) & 18.06 & $16.67(4.17-25.00)$ & 39.46 & 33.33 (25.00-50.00) & $<0.001$ \\
\hline
\end{tabular}

${ }^{*} P$ values were derived from Mann-Whitney $U$ tests

GM, geometric mean. 
Table 3 Binary logistic analysis examining associations with depressive symptoms*

\begin{tabular}{|c|c|c|c|c|c|c|}
\hline Variables & B & SE & Wald & $P$ value & OR & $95 \% \mathrm{Cl}$ \\
\hline Cognition domain (robust $\dagger$ ) & & & & & 1 & \\
\hline Limited & -0.242 & 0.099 & 5.973 & 0.015 & 0.785 & 0.647 to 0.953 \\
\hline Limited & 0.611 & 0.104 & 34.66 & $<0.001$ & 1.842 & 1.503 to 2.258 \\
\hline Self care domain (robust $\dagger$ ) & & & & & 1 & \\
\hline Getting along domain (robust $\dagger$ ) & & & & & 1 & \\
\hline Limited & 0.48 & 0.111 & 18.576 & $<0.001$ & 1.616 & 1.299 to 2.010 \\
\hline Life activities domain (robust $\dagger$ ) & & & & & 1 & \\
\hline Limited & 0.52 & 0.105 & 24.665 & $<0.001$ & 1.683 & 1.370 to 2.066 \\
\hline Participation (robust $\dagger$ ) & & & & & 1 & \\
\hline
\end{tabular}

*Adjusted gender, age, education level, employment status, living style, region, poverty, physical discomfort, chronic diseases, hospitalisation. †Reference group.

disability and depressive symptoms. Specifically, participants had problems in domains of participation, life activities, getting along, and mobility and had no problem in cognition domain had an increased risk of depressive symptoms. Furthermore, we identified the interaction between functional disability and demographic factors related to depressive symptoms for the first time.

\section{Prevalence of depressive symptoms}

In this study, we found that the prevalence of depressive symptoms was $52.94 \%$, which is the same as $53.8 \%$ reported in a cross-sectional survey in Shenzhen, southern China, ${ }^{36}$ but much higher than that of older adults in Korea (30.3\%) and Mexico (29.1\%). ${ }^{37}$ The

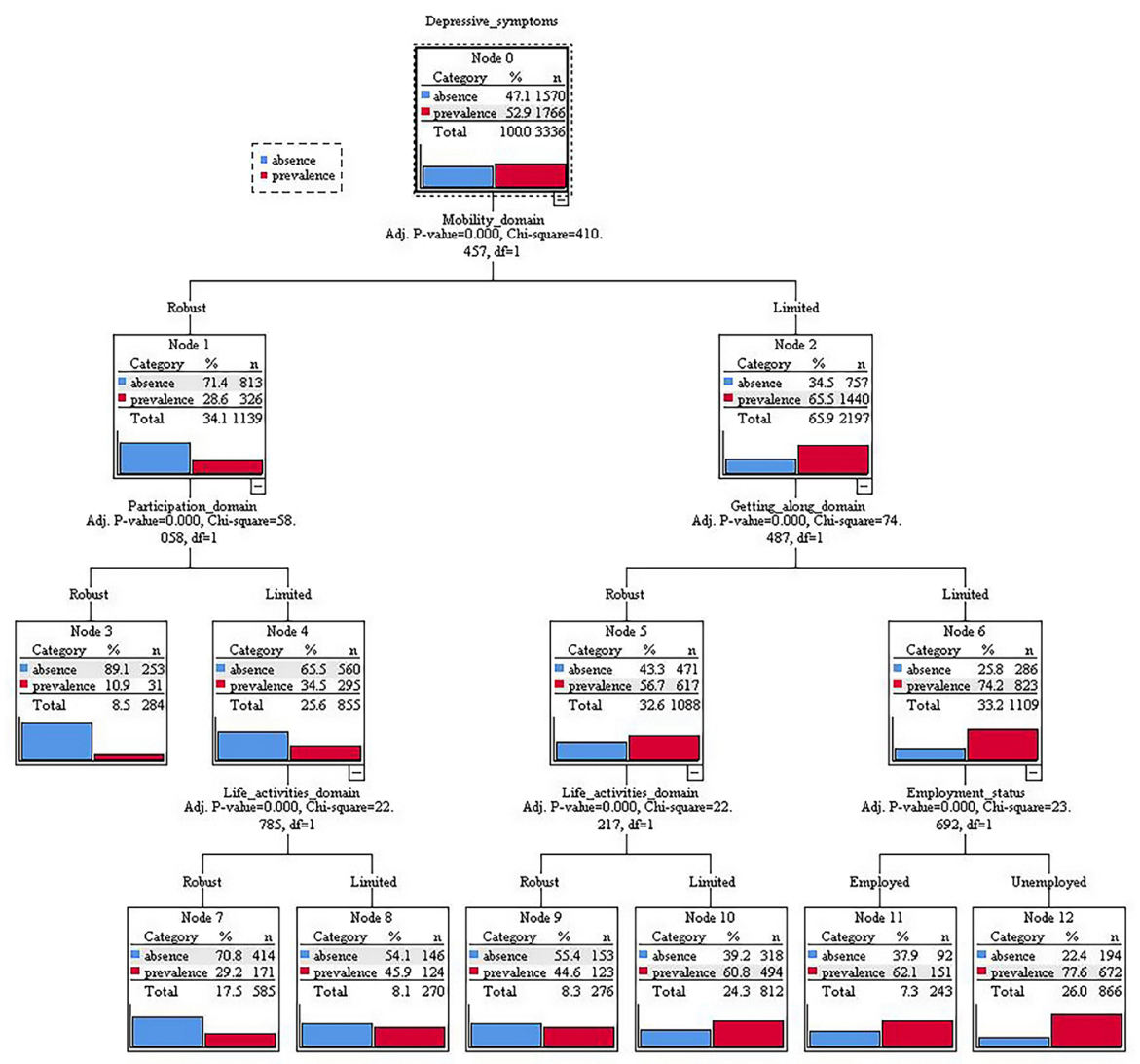

Figure 2 Classification and regression tree model $(\mathrm{N}=3336)$. 
heterogeneity of the prevalence among elderly people in different countries be because of differences of national culture and historical background as well as differences in survey samples of different assess scales and social support systems of older adults in different countries. This suggests that further population-based studies need to be performed in representative national samples.

\section{Association between functional disability and depressive symptoms}

We found that there were different results on the association between functional disability and depressive symptoms. This study found that there was a significant correlation between social participation and depressive symptoms and the risk of depressive symptoms was lower with no problem in social participation domain of older adults. Consistent with our research results, a study in Hong Kong found that a higher level of participation had a protective effect on depressive symptoms. ${ }^{39}$ A study in Japan found that the participation was significantly related to depressive symptoms, and living alone and low frequency of travel were risk factors for depressive symptoms in older adults. ${ }^{40}$ High participation can prevent the occurrence of depressive symptoms. ${ }^{41}$ As for the relationship between social participation and depressive symptoms, the possible reason is that older adults in rural China used to mainly engage in family-based agricultural activities and are basically idle at home when they are old. The main participation is leisure activities of WHODAS 2.0 questionnaire, such as contacts with friends and neighbours. Leisure activities can be a protective factor against depressive symptoms. ${ }^{42}$ Older adults can enjoy close communication, interaction, and companionship of neighbours and generate positive emotions, friendship and a sense of meaning and reduce their loneliness. These positive emotions can help prevent depressive symptoms in older adults. ${ }^{4344}$

We found that life activities was related to the presence of depressive symptoms, and after controlling for confounders, those who had problems in life activities had a higher risk of depressive symptoms. The results were similar to those of previous studies. Studies on older adults in Europe, America, the Middle East and other Asian countries have shown that the risk of depressive symptoms was related to the defect of living activities. ${ }^{45}$ Tomioka $e t a l^{46}$ conducted a survey in Japanese community and found that participation (such as community activities, voluntary activities) was related to the change of instrumental activities of daily living. Relatively complete mobility of physical activity (such as walking and riding) can affect the degree of participation. In the process of social participation, getting along and getting information (such as keeping healthy knowledge) among older adults can regulate their feelings toward negative life events, and then affect their mental health.

Interestingly, this study found that there was a negative correlation between cognition and depressive symptoms, that is, those with no problem in cognition were more prone to develop depressive symptoms. This is inconsistent with previous studies. Previous studies have found that the cognition of the elderly in developing countries was related to depressive symptoms. ${ }^{47}$ The two had some common clinical manifestations and risk factors, and depressive symptoms was an important risk factor for cognition and significantly increases the risk of cognitive impairment. ${ }^{48-50}$ However, Lou et $a \tilde{l}^{\tilde{1}}$ and Gale et $a \tilde{l}^{2}$ showed that there was no significant correlation between the cognition level and depressive symptoms in older adults. Considering the reasons, the old-age social security system in rural areas in China is imperfect. Financial support from their children is the main form of support available to older adults in rural areas, which is often unstable and unreliable. Older adults with normal cognition may have a clearer understanding of the pension problems, and may have more worries about their future life, which increases the psychological burden of the older adults and may lead to the occurrence of depressive symptoms.

\section{Interaction among variables}

In this study, the interaction between functional disability and demographic characteristics related to depressive symptoms was explored for the first time in Chinese population. Most studies have found that low educational level, ${ }^{32}$ female sex,$^{53}$ and increasing age ${ }^{54}$ were related to the occurrence of depressive symptoms in the elderly population. Simultaneously, some studies have noted the impact of functional disability on the mental health of the older adults. ${ }^{55}$ Although these analyses and the study of factors were meaningful, more comprehensive methods are needed to explore the degree to which the joint combination and interaction of related factors associated with depressive symptoms. In the current study, CART model was used to explore the interaction between functional disability and various variables. It was found that those with mobility problems, getting along problems, and were unemployed had the highest risk of depressive symptoms. Simultaneously, among who had no problems with mobility and participation, the incidence of depressive symptoms was the lowest. However, this interaction cannot be observed in binary logistic regression analysis, which further shows the advantages of CART model. We confirmed for the first time the interaction between functional disability and other demographic variables related to depressive symptoms in Chinese population. These results help understand which of the rural older adults are to develop depressive symptoms, and help design specific and comprehensive intervention strategies to improve the mental health level of them.

\section{Strengths and limitations}

Our study has several advantages. First, the sample response rate is high, and the large-scale survey results with high response rate are very robust. Second, we use the CART model in the method, which can overcome the possible colinearity between variables and automatically 
judge and classify them according to the significance of the test. It can not only explore the interaction between multivariables, but also be displayed by intuitive tree graph. Third, this is the first study to explore the interaction between functional disability and demographic factors related to depressive symptoms in the Chinese population, which provides more insights for the formulation of effective measures to prevent the occurrence of depressive symptoms.

However, this study also has some limitations. First, because of the characteristics of a cross-sectional study, inferring the causality between functional disability and depressive symptoms is limited, and the cohort study design needs to be considered in future studies. Second, the sample of this study comes from Anhui Province, China. Due to the heterogeneity of economic level and cultural background, the extension of the research results to other regions or other countries is limited. Finally, the sample of older adults depressive symptoms was determined by GDS-30, without the diagnosis of clinical professionals, and it may thus overestimate the true level of depressive symptoms.

\section{CONCLUSIONS}

In the rural older adults in Anhui Province, functional disability is significantly related to depressive symptoms, especially those with participation problems may face greater risk of depressive symptoms. Our study provides baseline information for future research and adds useful data on functional disability and depressive symptoms from rural China, which helps to better understand the epidemiology of geriatric depression in China and compares between China with other countries. At the same time, under the background of imperfect social support system in rural China, our findings will help relevant departments to formulate targeted intervention strategies to prevent depressive symptoms for the older adults. To be more specific, grassroots government should establish activity locations, set up leisure and entertainment facilities for the rural older adults, and establish mutual aid organisations to encourage rural older adults to communicate and engage in leisure and entertainment activities, so as to promote their mental health. At the same time, it is necessary to improve the security mechanism and improve the quality of life for them. In addition, rural communities should publicise and educate on health knowledge, introduce mental health clinics, establish health records for groups with high-risk depressive symptoms, and provide periodic mental health counselling services for those rural older adults, so as to improve the mental health level of and promote healthy ageing.

Contributors JR conceptualised the study. XW, YG and GC contributed to the study design, data collection and data processing and statistical analysis. XW and YG contributed to the literature review. JR wrote the article. JR, YG and HD revised the article. All authors reviewed the manuscript and approved the final manuscript. We thank the village committee members and village doctors who contributed to this study. $\mathrm{HD}$ is responsible for the overall content as guarantor.
Funding This research was funded by Research Projects of Humanities and Social Sciences in Colleges and Universities of Anhui Province under grant number SK2018A0165 and Doctoral Fund Project of Anhui Medical University under grant number XJ201545.

Disclaimer The funders had no role in the design of the study and collection, analysis, and interpretation of data and in writing the manuscript.

Competing interests None declared.

Patient consent for publication Not applicable.

Ethics approval This study was conducted in accordance with the Declaration of Helsinki and was approved by the Research Ethics Committee of Anhui Medical University (AMUREC: 2019H016).

Provenance and peer review Not commissioned; internally peer reviewed.

Data availability statement Data are available on reasonable request. Not applicable. The datasets generated during the study are not publicly available due to an ethical restriction but are available from the corresponding author on reasonable request.

Supplemental material This content has been supplied by the author(s). It has not been vetted by BMJ Publishing Group Limited (BMJ) and may not have been peer-reviewed. Any opinions or recommendations discussed are solely those of the author(s) and are not endorsed by BMJ. BMJ disclaims all liability and responsibility arising from any reliance placed on the content. Where the content includes any translated material, BMJ does not warrant the accuracy and reliability of the translations (including but not limited to local regulations, clinical guidelines, terminology, drug names and drug dosages), and is not responsible for any error and/or omissions arising from translation and adaptation or otherwise.

Open access This is an open access article distributed in accordance with the Creative Commons Attribution Non Commercial (CC BY-NC 4.0) license, which permits others to distribute, remix, adapt, build upon this work non-commercially, and license their derivative works on different terms, provided the original work is properly cited, appropriate credit is given, any changes made indicated, and the use is non-commercial. See: http://creativecommons.org/licenses/by-nc/4.0/.

ORCID iD

Hong Ding http://orcid.org/0000-0003-1448-1434

\section{REFERENCES}

1 National Bureau of Statistics. Statistical bulletin for national economic and social development, 2020. Available: http://www.stats. gov.cn/tjsj/zxfb/202002/t20200228_1728913.html [Accessed 15 Nov 2020].

2 World population ageing 2017 highlights. Available: https://www.un. org/en/development/desa/population/theme/ageing/WPA2017.shtml [Accessed 15 Nov 2020].

3 Huang $\mathrm{Y}$, Wang $\mathrm{Y}$, Wang $\mathrm{H}$, et al. Prevalence of mental disorders in China: a cross-sectional epidemiological study. Lancet Psychiatry 2019;6:211-24.

4 Fiske A, Wetherell JL, Gatz M. Depression in older adults. Annu Rev Clin Psychol 2009;5:363-89.

5 World Health Organization. Depression. Available: https://www.who. int/health-topics/depression\#tab=tab_1 [Accessed 15 Nov 2020].

6 Blazer DG. Depression in late life: review and commentary. J Gerontol A Biol Sci Med Sci 2003;58:M249-65.

7 World Health Organization. Depression and other common mental disorders: global health estimates. Geneva: World Health Organization, 2017.

8 Rong J, YH G, Meng NN. Prevalence rate of depression in Chinese elderly from 2010 to 2019: a meta-analysis. Chinese J EvidenceBased Med 2020;20:26-31.

9 Brandão DJ, Fontenelle LF, da Silva SA, et al. Depression and excess mortality in the elderly living in low- and middle-income countries: systematic review and meta-analysis. Int J Geriatr Psychiatry 2019;34:22-30.

10 Bock J-O, Hajek A, Weyerer S, et al. The impact of depressive symptoms on healthcare costs in late life: longitudinal findings from the AgeMooDe study. Am J Geriatr Psychiatry 2017;25:131-41.

11 Hall CA, Reynolds-lii CF. Late-life depression in the primary care setting: challenges, collaborative care, and prevention. Maturitas 2014;79:147-52.

12 Taheri Tanjanai P, Moradinazar M, Najafi F. Prevalence of depression and related social and physical factors amongst the Iranian elderly population in 2012. Geriatr Gerontol Int 2017;17:126-31. 
13 Ge L, Yap CW, Ong R, et al. Social isolation, loneliness and their relationships with depressive symptoms: a population-based study. PLoS One 2017;12:e0182145

14 Pino EC, Damus K, Jack B, et al. Adolescent socioeconomic status and depressive symptoms in later life: evidence from structural equation models. J Affect Disord 2018;225:702-8.

15 Ruiz M, Malyutina S, Pajak A, et al. Congruent relations between perceived neighbourhood social cohesion and depressive symptoms among older European adults: an East-West analysis. Soc Sci Med 2019;237:112454

16 Carter T, Morres ID, Meade O, et al. The effect of exercise on depressive symptoms in adolescents: a systematic review and metaanalysis. J Am Acad Child Adolesc Psychiatry 2016;55:580-90.

17 Guo Q, Bai X, Feng N. Social participation and depressive symptoms among Chinese older adults: a study on rural-urban differences. $J$ Affect Disord 2018;239:124-30.

18 Kong D, Solomon P, Dong X. Depressive symptoms and onset of functional disability over 2 years: a prospective cohort study. J Am Geriatr Soc 2019;67:S538-44.

19 Su P, Ding H, Zhang W, et al. The association of multimorbidity and disability in a community-based sample of elderly aged 80 or older in Shanghai, China. BMC Geriatr 2016;16:178.

20 World Health Organization. WHO global disability action plan 20142021: better health for all people with disability. Gevena, Switzerland: World Health Organization, 2015.

21 Hajjioui A, Abda N, Guenouni R, et al. Prevalence of disability in Morocco: results from a large-scale national survey. $J$ Rehabil Med 2019;51:805-12.

22 Hermsen LAH, Leone SS, Smalbrugge M, et al. Frequency, severity and determinants of functional limitations in older adults with joint pain and comorbidity: results of a cross-sectional study. Arch Gerontol Geriatr 2014;59:98-106.

23 Okoro CA, Hollis ND, Cyrus AC, et al. Prevalence of Disabilities and Health Care Access by Disability Status and Type Among Adults United States, 2016. MMWR Morb Mortal Wkly Rep 2018;67:882-7.

24 Lestari SK, Ng N, Kowal P, et al. Diversity in the factors associated with ADL-Related disability among older people in six middle-income countries: a Cross-Country comparison. Int J Environ Res Public Health 2019;16:1341.

25 Ćwirlej-Sozańska AB, Sozański B, Wiśniowska-Szurlej A, et al. An assessment of factors related to disability in ADL and IADL in elderly inhabitants of rural areas of south-eastern Poland. Ann Agric Environ Med 2018;25:504-11.

26 Kojima G. Frailty as a predictor of disabilities among communitydwelling older people: a systematic review and meta-analysis. Disabil Rehabil 2017;39:1897-908.

27 Nyunt MSZ, Lim ML, Yap KB, et al. Changes in depressive symptoms and functional disability among community-dwelling depressive older adults. Int Psychogeriatr 2012;24:1633-41.

28 Chao S-F. Functional disability and depressive symptoms: longitudinal effects of activity restriction, perceived stress, and social support. Aging Ment Health 2014;18:767-76.

29 Kilavuz A, Meseri R, Savas S, et al. Association of sarcopenia with depressive symptoms and functional status among ambulatory community-dwelling elderly. Arch Gerontol Geriatr 2018;76:196-201.

30 Yesavage JA, Brink TL, Rose TL, et al. Development and validation of a geriatric depression screening scale: a preliminary report. J Psychiatr Res 1982;17:37-49.

31 Dong Q, Liu J-J, Zheng R-Z, et al. Obesity and depressive symptoms in the elderly: a survey in the rural area of Chizhou, Anhui Province. Int J Geriatr Psychiatry 2013;28:227-32.

32 Rong J, Chen G, Wang X, et al. Correlation between depressive symptoms and quality of life, and associated factors for depressive symptoms among rural elderly in Anhui, China. Clin Interv Aging 2019;14:1901-10.

33 Üstun TB, Ebrary I. Measuring health and disability: manual for who disability assessment schedule WHODAS 2.0. Geneva: WHO, 2010

34 World Health Organization. International classification of functioning, disability and health (ICF). Geneva: WHO, 2001.

35 Machuca C, Vettore MV, Krasuska M, et al. Using classification and regression tree modelling to investigate response shift patterns in dentine hypersensitivity. BMC Med Res Methodol 2017;17:120.
36 Dong X, Zhao L, Sun T, et al. Prevalence of depressive symptoms and associated factors among internal migrants with tuberculosis: a cross-sectional study in China. Am J Trop Med Hyg 2020;102:31-5.

37 Park J-I, Park TW, Yang J-C, et al. Factors associated with depression among elderly Koreans: the role of chronic illness, subjective health status, and cognitive impairment. Psychogeriatrics 2016:16:62-9.

38 Ortiz GG, Arias-Merino ED, Flores-Saiffe ME, et al. Prevalence of cognitive impairment and depression among a population aged over 60 years in the metropolitan area of Guadalajara, Mexico. Curr Gerontol Geriatr Res 2012;2012:175019

39 Lou VWQ, Chi I, Kwan CW, et al. Trajectories of social engagement and depressive symptoms among long-term care facility residents in Hong Kong. Age Ageing 2013;42:215-22.

40 Kuroda A, Tanaka T, Hirano H, et al. Eating alone as social disengagement is strongly associated with depressive symptoms in Japanese community-dwelling older adults. J Am Med Dir Assoc 2015;16:578-85.

41 Lee J, Jang S-N, Cho S-I. Gender differences in the trajectories and the risk factors of depressive symptoms in later life. Int Psychogeriatr 2017:29:1495-505.

42 Nagata S, McCormick B, Piatt J. Leisure behavior and sense of coherence in the context of depression. $J$ Community Psychol 2020;48:283-301.

43 van den Brink RHS, Schutter N, Hanssen DJC, et al. Prognostic significance of social network, social support and loneliness for course of major depressive disorder in adulthood and old age. Epidemiol Psychiatr Sci 2018;27:266-77.

44 Zahodne LB, Watson CW-M, Seehra S, et al. Positive psychosocial factors and cognition in ethnically diverse older adults. $J$ Int Neuropsychol Soc 2018;24:294-304.

45 Kilzieh N, Rastam S, Ward KD, et al. Gender, depression and physical impairment: an epidemiologic perspective from Aleppo, Syria. Soc Psychiatry Psychiatr Epidemiol 2010;45:595-602.

46 Tomioka K, Kurumatani N, Hosoi H. Association between social participation and 3-year change in instrumental activities of daily living in community-dwelling elderly adults. J Am Geriatr Soc 2017;65:107-13.

47 Del Brutto OH, Mera RM, Del Brutto VJ, et al. Influence of depression, anxiety and stress on cognitive performance in community-dwelling older adults living in rural Ecuador: results of the Atahualpa project. Geriatr Gerontol Int 2015;15:508-14.

48 Ismail Z, Smith EE, Geda Y, et al. Neuropsychiatric symptoms as early manifestations of emergent dementia: provisional diagnostic criteria for mild behavioral impairment. Alzheimers Dement 2016;12:195-202.

49 Lara E, Koyanagi A, Domènech-Abella J, et al. The impact of depression on the development of mild cognitive impairment over 3 years of follow-up: a population-based study. Dement Geriatr Cogn Disord 2017;43:155-69.

50 Youn H, Lee ES, Lee S, et al. Regional glucose metabolism due to the presence of cerebral amyloidopathy in older adults with depression and mild cognitive impairment. J Affect Disord 2018;239:30-6.

51 Lou CY, KY W, Shen S. Investigation and analysis of depression and cognitive status of elderly people in aged care institutions in urban area of Hangzhou. Chin J Mod Nurs 2019;25:72-6.

52 Gale CR, Allerhand M, Deary IJ, et al. Is there a bidirectional relationship between depressive symptoms and cognitive ability in older people? A prospective study using the English longitudinal study of ageing. Psychol Med 2012;42:2057-69.

53 Hernández-Vásquez A, Vargas-Fernández R, Bendezu-Quispe G, et al. Depression in the Peruvian population and its associated factors: analysis of a national health survey. $J$ Affect Disord 2020;273:291-7.

54 Xiong N, Liu Q, Lv X, et al. Demographic and psychosocial variables could predict the occurrence of major depressive disorder, but not the severity of depression in patients with First-episode major depressive disorder in China. J Affect Disord 2020;274:103-11.

55 Xiang $X$, Yang Y, Cheng J. The impact of late-life disability spectrum on depressive symptoms: a Fixed-Effects analysis of panel data. J Gerontol B Psychol Sci Soc Sci 2020:gbaa060. 